\title{
Measuring Outcomes in Psoriatic Arthritis: Comparing Routine Assessment of Patient Index Data and Psoriatic Arthritis Impact of Disease
}

\author{
Jessica A. Walsh ${ }^{1}$ (D), Marilyn T. Wan², Christine Willinger ${ }^{3}$, M. Elaine Husni ${ }^{4}$ (D) Jose U. Scher ${ }^{5}$ (D), \\ Soumya M. Reddy ${ }^{5}$, and Alexis Ogdie ${ }^{1}$ (D)
}

\begin{abstract}
Objective. To examine the construct validity of Routine Assessment of Patient Index Data 3 (RAPID3) and Psoriatic Arthritis Impact of Disease (PsAID) in patients with psoriatic arthritis (PsA). In examining construct validity, we also addressed scores among subgroups with severe psoriasis, polyarticular disease, enthesitis, and dactylitis, and evaluated influences of sociodemographic factors and comorbidities (contextual factors) on these patient-reported outcomes (PRO).

Methods. Patients with PsA were enrolled in the Psoriatic Arthritis Research Consortium (PARC) between 2014 and 2016. PARC is a longitudinal observational cohort study conducted at 4 US institutions. In this cross-sectional study, construct validity was assessed by examining Spearman correlation coefficients for RAPID3 and PsAID with physician-reported disease activity measures and other PRO [e.g., Medical Outcomes Study Short Form-12 physical component summary/mental component summary (SF-12 PCS/ MCS), Functional Assessment of Chronic Illness Therapy-Fatigue scale (FACIT-F)]. Contextual factors and disease subgroups were assessed in multivariable linear regression models with RAPID3 or PsAID12 as outcomes of interest and the hypothesized contextual factors as covariates.

Results. Among 401 patients enrolled in PARC, 347 completed RAPID3 or PsAID12. Of these, most were white females with a mean age of 51.7 years (SD 14.02). RAPID3 and PsAID were highly correlated $(r=0.90)$. These measures were also correlated with the SF-12 PCS $(r=-0.67)$ and FACIT-F $(r=-0.77)$. Important contextual factors and disease subgroups included enthesitis, joint counts, education, insurance type, and depression.

Conclusion. RAPID3 and PsAID12 have excellent construct validity in PsA and are strongly correlated despite differing items. Contextual factors (i.e., the presence of depression and obesity) should be considered when interpreting raw scores of the RAPID3 and PsAID12.
\end{abstract}

Key Indexing Terms: patient-reported outcomes, psoriatic arthritis

Understanding patient perceptions of health and function are necessary for optimizing clinical decision making. Patient-reported outcomes (PRO) provide valuable insights into

Supported by the US National Institutes of Health/ US National Institute of Arthritis and Musculoskeletal and Skin Diseases (R01 AR072363).

$D r$. Walsh has received consulting fees or research grants from AbbVie and Pfizer. Dr. Husni is a coinventor on a patent for a psoriatic arthritis questionnaire (Psoriatic Arthritis Screening Evaluation), for which she receives royalties. Dr. Scher has received consulting fees from Novartis. Dr. Ogdie has received grants from Amgen and Pfizer to the trustees of the University of Pennsylvania.

${ }^{1} J$.A. Walsh, MD, MBA, A. Ogdie, MD, MSCE, Division of Rheumatology, University of Pennsylvania Perelman School of Medicine, Philadelphia; ${ }^{2}$ M.T. Wan, MBChB, MPH, Department of Dermatology, University of Pennsylvania Perelman School of Medicine, Philadelphia; ${ }^{3} C$. Willinger, BS, University of Pennsylvania, Philadelphia, Pennsylvania; ${ }^{4}$ M.E. Husni, MD, MPH, Cleveland Clinic, Cleveland, Ohio; J.U. Scher, MD S, S.M. Reddy $M D^{5}$, New York University School of Medicine, New York, New York, USA. Address correspondence to Dr. A. Ogdie, Division of Rheumatology, Perelman School of Medicine, University of Pennsylvania, Philadelphia, PA 19104, USA.Email:alexis.ogdie@uphs.upenn.edu.

Accepted for publication September 18, 2019. patients' perceptions of their disease and individual priorities for improvements. PRO also communicate information that may be difficult to ascertain with standard provider assessments, particularly with heterogeneous diseases such as psoriatic arthritis (PsA). Because patient perceptions of health are important targets for intervention, it is necessary to select and understand appropriate PRO for assessments of PsA ${ }^{1,2}$. Selection of PRO for PsA is challenging. There are a large number of available PRO measuring overlapping but distinct aspects of disease. Time and resources for both patients and clinicians limit the number of PRO that can be included in studies and daily clinical practice. Further, when too many PRO are required from patients, questionnaire fatigue erodes the quality of data ${ }^{3}$.

For the purposes of clinical practice and longitudinal cohort studies, several PRO are attractive for various reasons. The Routine Assessment of Patient Index Data 3 (RAPID3) is appealing because it is widely recognized and feasible and is largely considered the most commonly used PRO in the United States across inflammatory diseases ${ }^{4,5,6}$. Further, RAPID3 has been validated as a measure of disease activity in rheumatoid arthritis (RA), ankylosing spondylitis, and more recently

Personal non-commercial use only. The Journal of Rheumatology Copyright @ 2020 . All rights reserved. 
PsA $^{7,8,9,10,11}$. This means RAPID3 can be useful when comparing between inflammatory arthritis subsets, studying multiple types of inflammatory arthritis in aggregate, and seeing patients with varied diagnoses in a single clinic session. Unlike the RAPID3, initially designed for patients with RA, the Psoriatic Arthritis Impact of Disease (PsAID) questionnaire was specifically developed for PsA by patients with PsA and each question is specifically attributed to PsA (i.e., each item, such as fatigue, is followed by the words "due to your PsA") ${ }^{12,13,14}$. It was designed to broadly measure the effect of disease on diverse aspects of patients' lives and has also been shown to correlate strongly with disease activity measures, such as Disease Activity in PSoriatic Arthritis $(D A P S A)^{15}$, and disease activity cutoff scores have been established, including a Patient Acceptable Symptom State ${ }^{12}$. Further, a principal component analysis identified a subset of questions within the PsAID12 that represented the effect of cutaneous psoriasis. Both RAPID3 and PsAID12 are among the most feasible PRO and are widely used and accepted by patients and researchers ${ }^{16,17}$. Many other PRO also exist; most of these more narrowly measure concepts such as fatigue or work productivity, or broadly measure health-related quality of life (HRQOL) ${ }^{4}$.

While RAPID3 and PsAID performed well in PsA overall, there are several common demographic and comorbid factors that influence PRO. In general, women with PsA report higher self-assessed disease activity scores compared to men ${ }^{18,19,20,21,22}$. Additionally, obesity has been associated with less favorable PRO in patients with axial spondyloarthritis (axSpA) 23,24,25,26. The influence of contextual factors, such as sociodemographics and comorbidities, on PRO is not well understood in PsA ${ }^{22,27,28,29}$. To inform PRO selection and interpretation, the objectives of our study were to characterize the construct validity of RAPID3 and PsAID in the general PsA population and in subsets with polyarticular disease ( 4 or more active joints), enthesitis, and dactylitis; and to evaluate influences of common sociodemographic factors and comorbidities on these 2 PRO.

\section{MATERIALS AND METHODS}

Patients and study population. Psoriatic Arthritis Research Consortium (PARC) is a longitudinal cross-sectional observational cohort study that includes 4 institutions in the US: the University of Pennsylvania (Penn Psoriatic Arthritis Program), Cleveland Clinic (Arthritis and Musculoskeletal Center), New York University (Psoriatic Arthritis Center), and the University of Utah (Utah Spondyloarthritis Program). The 4 centers in PARC standardized data collection and developed a Research Electronic Data Capture, or REDCap, database with a common data model. The study period was 2014 to 2016. Patients were 18 years or older and met ClASsification for Psoriatic ARthritis (CASPAR) criteria ${ }^{30}$. Only baseline data are included in this analysis.

Physician-assessed variables collected. Baseline demographics and social histories that were reported by participants included age, sex, race, ethnicity, body mass index (BMI), smoking status (ever vs never), current alcohol use (yes/no), highest level of education, insurance type, work status, and income level. Additional information obtained by physicians through a history and record review included location of study center, duration of PsA (since diagnosis), duration of psoriasis (since diagnosis), fulfillment of Assessment of Spondyloarthritis international Society (ASAS) criteria for axSpA, inflammatory back pain as defined by ASAS criteria or sacroiliitis as defined by joint imaging ${ }^{31}$, and comorbidities including presence of obesity (BMI $>30 \mathrm{~kg} / \mathrm{m}^{2}$ ), fibromyalgia, concurrent osteoarthritis, cardiovascular disease, and diabetes. Physician examination measures included tender joint counts (TJC) and swollen joint counts (SJC; 66/68 at 3 centers and 76/78 at the fourth center), enthesitis count $>0$ (measured by Leeds Enthesitis Index ${ }^{32}$ plus bilateral plantar fascia insertions), dactylitis count $>0$, and typical psoriatic nail dystrophy (onycholysis, pitting, or hyperkeratosis) ${ }^{30}$. Physicians also reported their global assessments for cutaneous psoriasis, arthritis, and overall health. Data were collected at a single timepoint at enrollment in this cross-sectional study. If more than 1 follow-up was available, only the enrollment visit was included.

PRO. All sites collected the RAPID3 [1 site collected the Health Assessment Questionnaire-Disability Index (HAQ-DI), patient pain, and patient global (range $0-10)^{33}$, and these scores were transformed to RAPID3 scores per a previously described algorithm $]^{34}$. In addition, 3 sites collected the Medical Outcomes Study Short Form-12 physical component summary (SF-12 PCS); the physical component score on the SF-12 is highly correlated with the SF-36 PCS used in PsA clinical trials ${ }^{35}$. Two sites additionally collected the PsAID12, the Bath Ankylosing Spondylitis Disease Activity Index (BASDAI), the Functional Assessment Of Chronic Illness Therapy-Fatigue scale (FACIT-F), Dermatology Life Quality Index, Work Limitations Questionnaire (WLQ), and Work Productivity and Activity Impairment. Permission from the FACIT owners was obtained for the FACIT-F. Licenses have been obtained for the use of the SF-12 and the WLQ from Optum and Tufts University, respectively. Patients also reported insurance status, work status, education level, smoking, alcohol consumption, and a basic set of comorbidities including depression (defined as patient-reported depression or Patient Health Questionnaire-9 score > 10). Construct validity - a priori hypotheses. Construct validity is the ability of an instrument to measure the concepts it attempts to measure ${ }^{36}$. Convergent validity, one aspect of construct validity, is the concept that 2 measures that should be related to each other are, in fact, related. We believe that both RAPID3 and PsAID are addressing the construct of "disease impact" or how much the disease is currently affecting the patient's life. We hypothesized $a$ priori that RAPID3 and PsAID-9 and - 12 would be strongly correlated (rho 0.8-1.0; Supplementary Table 1, available with the online version of this article $)^{37}$. Further, we hypothesized that these outcomes would have strong correlations with physical function measures (i.e., SF-12 PCS) and other disease effect measures (BASDAI), and moderate correlation (rho 0.6-0.8) with fatigue, HRQOL assessments, work limitations, and swollen and TJC. While we hypothesized that depression would affect the total score (as a contextual factor), we predicted that there would be a low correlation between RAPID3 and SF-12 mental component summary (MCS). Additionally, we hypothesized that RAPID3 and PsAID would differentiate well between those with low and high disease activity when stratified by swollen and TJC, enthesitis, and dactylitis (PsA subgroups). Finally, we hypothesized that there would be associations between the RAPID3 and PsAID scores with contextual factors (including obesity, older age, and female sex; comorbidities such as hypertension, diabetes, and depression; and socioeconomic status $)^{38}$.

Statistical analysis. We first descriptively reported the study population characteristics. We assessed convergent validity by examining the Spearman correlation (given a skewed distribution of data) for RAPID3 and PsAID12 with the other PRO and physician-reported disease activity measures (as described above). Another form of construct validity is the ability to differentiate between known groups or differing disease states ${ }^{39,40,41}$. We used swollen joints as a reference standard. We plotted each PRO and the number of swollen joints. We compared the mean score for RAPID3 and PsAID12 among patients with 4 or more swollen joints versus 3 or fewer swollen joints (this cutoff was chosen because it is typically used for eligibility into PsA randomized controlled trials).

Personal non-commercial use only. The Journal of Rheumatology Copyright $\subset$ 2020. All rights reserved. 
Contextual factors were investigated as confounders. These factors (e.g., demographic or clinical characteristics) result in differential scoring of the questionnaire of interest. A list of hypothesized or potential contextual factors was developed prior to statistical testing and included sex, age ( $<50$ or $\geq 50$ yrs, according to median split), race, education level, insurance type, work status, depression, obesity, diabetes, or cardiovascular disease. We examined the association between potential contextual factors and RAPID3 and PsAID12 scores by plotting the median RAPID3 and PsAID12 scores by subgroup of the potential contextual factor. We then used univariable linear regression models to identify the statistical association of potential contextual factors with the RAPID3 and subsequently formed multivariable linear regression models in a purposeful selection method (testing only biologically relevant variables and including only those variables significant at the univariable stage in a full model, defined as $\mathrm{p}<0.05)$ to determine which factors continued to have an independent relationship with the RAPID3 score when accounting for the other factors in the model. This modeling process was repeated for PsAID12. We used a complete case design in this cross-sectional study.

Ethics approval. Institutional Review Board approval was obtained at all 4 sites: University of Pennsylvania (PARC-819801; PARC-B- 828357), Cleveland Clinic (IRB 07-623), New York University (14-00487), and University of Utah (IRB_00074499). Patients signed a written consent form prior to enrollment in the study.

\section{RESULTS}

The study enrolled 401 patients among the 4 centers. After excluding patients who did not complete the RAPID3 or PsAID12, 347 patients remained. The cohort is representative of patients with PsA in the United States: $53.6 \%$ female, mean age 51.7 years (SD 14.02), and mostly white (Table 1 ). The prevalences of obesity and depression in the cohort were $42 \%$ and $19 \%$, respectively. Other common comorbidities in the cohort were diabetes (10\%) and cardiovascular disease (18\%). Using RAPID3 categories for disease activity designed for RA, the mean disease activity was moderate ${ }^{33}$. The mean PsAID12 score was 3.24 (SD 2.38), and 130 patients (63\%) were below the published patient acceptable symptom state of $4^{12}$.

RAPID3 and PSAID12 construct validity. We assessed the correlation among the PRO and the provider assessments (Figure 1 and Table 2). The RAPID3 was strongly correlated with the PsAID12 $(r=0.90, p<0.001$; this was identical for PsAID9; Figure 1). The magnitude of correlation between RAPID3 and the other PRO was similar to the magnitude of correlation between PsAID12 and the other PRO. Specifically, BASDAI strongly correlated with both RAPID3 $(r=0.88)$ and PsAID12 $(\mathrm{r}=0.87)$. Correlations were also moderate to strong with FACIT-F for RAPID3 $(\mathrm{r}=-0.77)$ and PsAID12 $(\mathrm{r}=-0.84)$. Correlations were modest to moderate between RAPID3 and SF-12 PCS, SF-12 MCS, WLQ, physician global assessment (PGA), physician assessment of arthritis, SJC, and TJC (range: $r=0.39-0.67$ ). Similarly, correlations were modest to moderate between PsAID12 and SF-12 PCS, SF-12 MCS, WLQ, PGA, physician assessment of arthritis, SJC, and TJC (range: rho $=0.37-0.73$ ).

To assess the ability of these measures to distinguish between known groups, we examined a series of different cutpoints in the SJC. There was a significant difference among those with 4 or
Table 1. Characteristics of patients with RAPID3 or PsAID12 data $(\mathrm{n}=$ $347)^{*}$.

\begin{tabular}{|c|c|}
\hline Characteristics & Values \\
\hline Age, yrs, mean (SD) & $51.7(14.0)$ \\
\hline \multicolumn{2}{|l|}{ Sex } \\
\hline Female, n (\%) & $186(53.6)$ \\
\hline \multicolumn{2}{|l|}{ Race, n (\%) } \\
\hline White & $300(86.5)$ \\
\hline Black & $6(1.7)$ \\
\hline Native American & $2(0.6)$ \\
\hline Asian & $12(3.5)$ \\
\hline Other & $13(3.8)$ \\
\hline Unknown & $7(2.0)$ \\
\hline BMI, mean (SD) & $29.2(6.7)$ \\
\hline \multicolumn{2}{|l|}{ Ethnicity } \\
\hline Hispanic, n (\%) & $7(2.0)$ \\
\hline \multicolumn{2}{|l|}{ Center, $\mathrm{n}(\%)$} \\
\hline Cleveland Clinic & $91(26.2)$ \\
\hline New York University & $47(13.5)$ \\
\hline University of Utah & $148(42.7)$ \\
\hline University of Pennsylvania & $61(17.6)$ \\
\hline \multicolumn{2}{|l|}{ Current therapies, $\mathrm{n}(\%)^{\dagger}$} \\
\hline Methotrexate & $114(32.9)$ \\
\hline Leflunomide & $17(4.9)$ \\
\hline Sulfasalazine & $19(5.5)$ \\
\hline Apremilast & $14(4.0)$ \\
\hline Adalimumab & $61(17.6)$ \\
\hline Etanercept & $67(19.3)$ \\
\hline Certolizumab & $3(0.9)$ \\
\hline Golimumab & $9(2.6)$ \\
\hline Infliximab & $20(5.8)$ \\
\hline Ustekinumab & $16(4.6)$ \\
\hline Secukinumab & $2(0.6)$ \\
\hline HCQ & $6(1.7)$ \\
\hline Any nbDMARD or bDMARD & $231(66.6)$ \\
\hline \multicolumn{2}{|l|}{ Joint counts, mean $(S D)^{\wedge}$} \\
\hline 66 swollen & $2.2(3.8)$ \\
\hline 68 tender & $4.3(7.2)$ \\
\hline 76 swollen & $4.5(11.2)$ \\
\hline 78 tender & $7.3(12.5)$ \\
\hline \multicolumn{2}{|l|}{ PGA, mean (SD) } \\
\hline Overall & $3.4(2.2)$ \\
\hline Arthritis & $3.0(2.2)$ \\
\hline Skin & $2.0(2.2)$ \\
\hline $\mathrm{PtGA}^{* *}$, mean $(\mathrm{SD})$ & $3.7(2.7)$ \\
\hline Pain assessment, mean (SD) & $4.0(2.8)$ \\
\hline HAQ score, mean $(\mathrm{SD})$ & $1.6(1.7)$ \\
\hline RAPID3, mean (SD) & $8.6(6.1)$ \\
\hline PsAID9*, mean (SD) & $3.4(2.4)$ \\
\hline PsAID $12^{*}$, mean (SD) & $3.2(2.4)$ \\
\hline
\end{tabular}

* PsAID was completed by 208 patients; RAPID3 was completed by 341 patients. ${ }^{* *}$ The global assessments use the $0-10$ range and were taken from the RAPID3. ${ }^{\dagger}$ Some patients were receiving concomitant therapy; percentages do not sum up to 100 . $^{\wedge}$ One center collected only the $76 / 78$ joint count. BMI: body mass index; HAQ: Health Assessment Questionnaire; nbDMARD: nonbiologic disease-modifying antirheumatic drug; bDMARD: biologic DMARD; RAPID3: Routine Assessment of Patient Index Data, range 0-30; HCQ: hydroxychloroquine; PsAID: Psoriatic Arthritis Impact of Disease questionnaire; PGA: physician global assessments; PtGA: patient global assessments. 

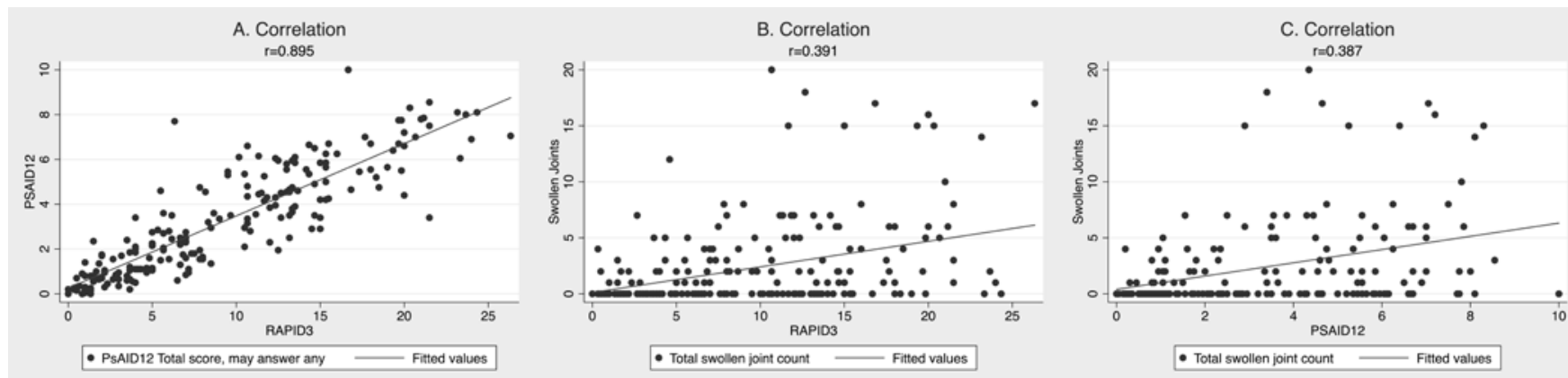

Figure 1. Construct validity: correlation of RAPID, PsAID, and SJC. Correlation between (A) RAPID3 and PsAID12, (B) RAPID3 and SJC (0-66), and (C) PsAID12 and SJC (0-66). RAPID3 and PsAID are strongly correlated whereas either measure has only moderate correlation with the SJC. RAPID3: Routine Assessment of Patient Index Data 3; PsAID12: Psoriatic Arthritis Impact of Disease 12; SJC: swollen joint count.

Table 2. Correlation among instruments in a cross-sectional setting.

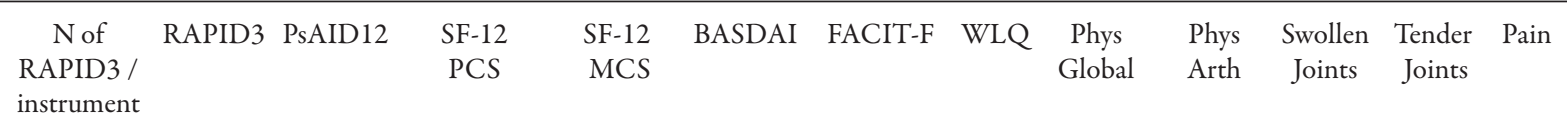

\begin{tabular}{|c|c|c|c|c|c|c|c|c|c|c|c|c|c|}
\hline PsAID12 & 207 & $0.90^{*}$ & & & & & & & & & & & \\
\hline PsAID9 & 207 & $0.90^{*}$ & $1.00^{*}$ & & & & & & & & & & \\
\hline SF-12 PCS & 270 & $-0.67^{*}$ & $-0.73^{*}$ & & & & & & & & & & \\
\hline SF-12 MCS & 270 & $-0.41^{*}$ & $-0.48^{*}$ & 0.11 & & & & & & & & & \\
\hline FACIT-F & 188 & $-0.77^{*}$ & $-0.84^{*}$ & $0.71^{*}$ & $0.54^{*}$ & $-0.78^{*}$ & & & & & & & \\
\hline WLQ $^{* *}$ & 141 & $0.67^{*}$ & $0.72^{*}$ & $-0.63^{*}$ & $-0.50^{*}$ & $0.68^{*}$ & $-0.76^{*}$ & & & & & & \\
\hline Phys Global & 304 & $0.63^{*}$ & $0.63^{*}$ & $-0.50^{*}$ & $-0.26^{*}$ & $0.56^{*}$ & $-0.48^{*}$ & $0.45^{*}$ & & & & & \\
\hline Tender Joints & 231 & $0.42^{*}$ & $0.37^{*}$ & $-0.33^{*}$ & $-0.15^{*}$ & $0.35^{*}$ & $-0.28^{*}$ & $0.29^{*}$ & $0.55^{*}$ & $0.63^{*}$ & $0.77^{*}$ & & \\
\hline Pain & 344 & $0.94^{*}$ & $0.89^{*}$ & $-0.63^{*}$ & $-0.34^{*}$ & 0.85 & -0.72 & 0.62 & 0.64 & 0.61 & 0.39 & 0.41 & \\
\hline Global & 343 & $0.93^{*}$ & $0.83^{*}$ & $-0.62^{*}$ & $-0.42^{*}$ & 0.81 & -0.72 & 0.64 & 0.56 & 0.55 & 0.35 & 0.38 & $0.83^{*}$ \\
\hline
\end{tabular}

Spearman correlation coefficients are shown here. Yellow indicates a strong correlation (rho $0.8-1.0)$ and blue a moderate correlation $(0.6-0.79) .{ }^{*} \mathrm{p}<0.05$. ** The productivity score of the Work Limitations Questionnaire (WLQ) is shown here. The WLQ index had nearly identical coefficients. Numbers in italics: Pain and global assessment are part of the RAPID3, so the correlation is expected to be high. Note that the additional columns with the other outcomes were not part of the original hypotheses. RAPID3: Routine Assessment of Patient Index Data 3; PsAID12: Psoriatic Arthritis Impact of Disease 12-question version; PsAID9: Psoriatic Arthritis Impact of Disease 9-question version; SF-12 PCS: Medical Outcomes Study Short Form-12 physical component summary; SF-12 MCS: SF-12 mental component summary; BASDAI: Bath Ankylosing Spondylitis Disease Activity Index; FACIT-F: Functional Assessment of Chronic Illness Therapy Fatigue scale; Phys global and Phys arth: physician global assessments for overall health and arthritis, respectively.

more swollen joints compared to those with 3 or fewer swollen joints (PsAID12 $\mathrm{n}=183$; RAPID3 $=231 ; P<0.001$; Figure 2) and similarly a significant difference $(P<0.001)$ when we examined $0-1$ swollen joints compared to 2 or more. Similarly, RAPID3 differentiated between patients with and without enthesitis on examination $(P<0.01$, Figure 3$)$; however, PsAID was not significantly different between patients with and without enthesitis.

Contextual factors and PsA subgroups. In Table 3, we present univariable and multivariable associations with the RAPID3 score. These were significantly associated with the RAPID3 score in univariable models: sex, alcohol use, center, BMI, education level, insurance type, SJC, TJC, enthesitis count, depression, and obesity. In a multivariable model, we found significant associations with RAPID3 in postgraduate education, insurance type, enthesitis, and TJC. Sex was no longer significant after accounting for these other factors. Removing it from the model did not change the point estimates or CI of the other elements and did not significantly affect the $\mathrm{R}^{2}$ value or Akaike information criteria. We also examined univariable and multivariable associations with the PsAID12 in Supplementary Table 2 (available with the online version of this article). In a multivariable model, we found that insurance type, enthesitis, SJC, and depression were significantly associated with PsAID12.

\section{DISCUSSION}

In this cross-sectional study, we demonstrated the construct validity of RAPID3 and PsAID12 in PsA; they are strongly associated with each other and correlate well with measures of similar constructs, replicating a priori hypotheses. To our knowledge, this is the first study to directly compare RAPID3 and 

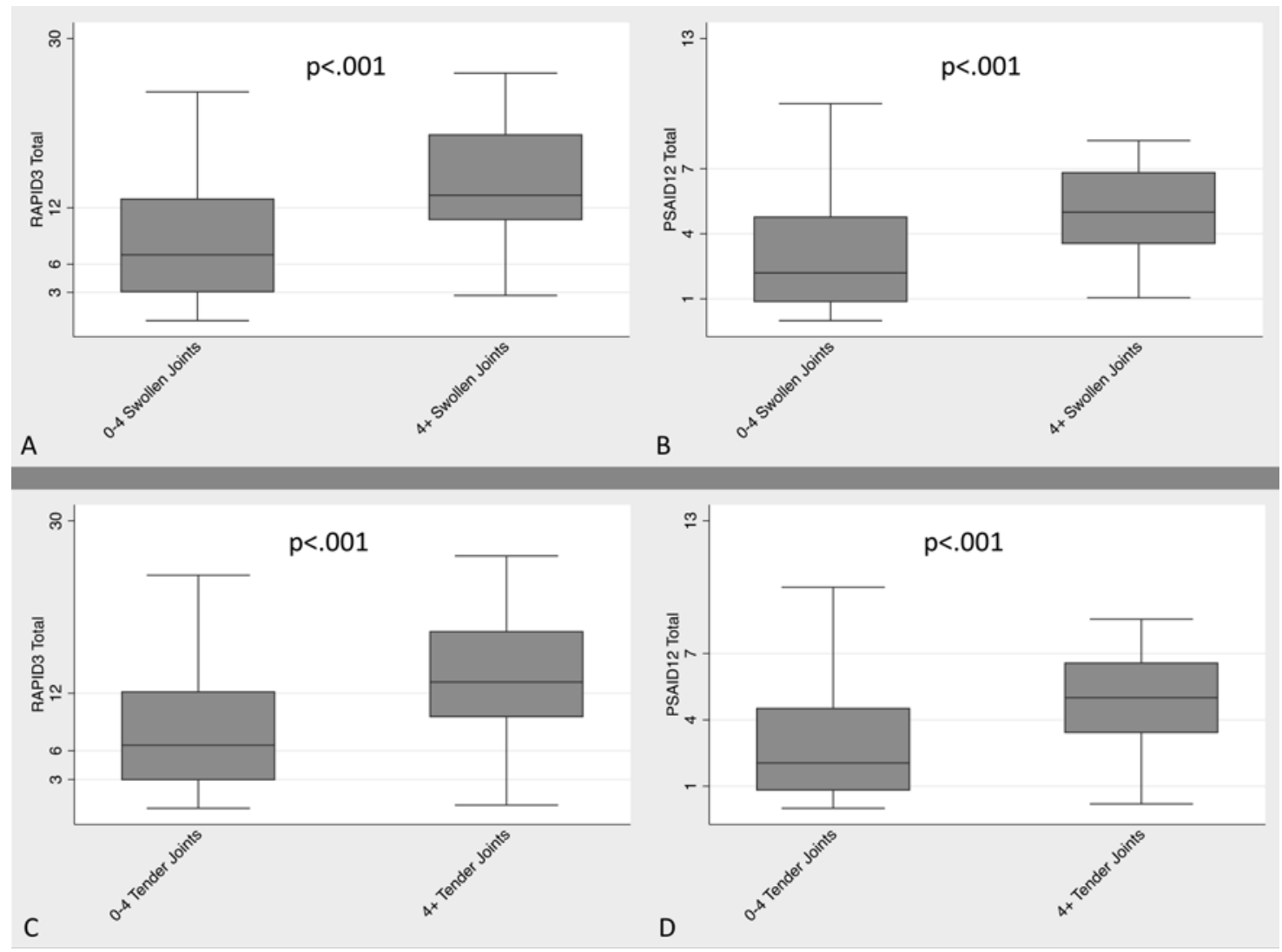

Figure 2. Known groups: RAPID3 and PsAID by SJC and by tender joint count. (A) RAPID3 and (B) PsAID12 scores are significantly different among patients with low disease activity (defined by SJC < 4) compared to those with 4 or more swollen joints, suggesting that these measures differentiate between low and higher disease activity states. (C) RAPID3 and (D) PsAID12 scores are significantly different among patients with low disease activity (defined by a tender joint count $<4$ ) compared to those with 4 or more tender joints, suggesting that these measures differentiate between low and higher disease activity states. RAPID3: Routine Assessment of Patient Index Data 3; PsAID12: Psoriatic Arthritis Impact of Disease 12; SJC: swollen joint count.

PsAID and we found that despite having different items on the questionnaires, they are highly correlated and measure the same construct: disease impact. Both RAPID3 and PsAID differentiated well between high and low disease states as demonstrated by SJC and TJC. The presence of enthesitis also seemed to be identified by the RAPID3 because patients with enthesitis had a higher score than patients without enthesitis, and it remained in the multivariable model after adjustment for other factors.

RAPID3 is a "multidimensional" instrument that involves questions not only about functional ability but also pain and includes a "global" assessment that outlines the patient's overall status ${ }^{33}$. Previous studies in RA have shown that RAPID3 correlates well with the 28-joint count Disease Activity Score (a measure of peripheral arthritis disease activity), the patient pain assessment, and inflammatory biomarkers (i.e., erythrocyte sedimentation rate or $\mathrm{C}$-reactive protein) and thus has construct validity in $\mathrm{RA}^{42}$. However, unlike in RA, in our study the RAPID3 was only mildly correlated with disease activity (i.e., SJC and TJC). Our results better support RAPID3 as a measure of disease effect rather than disease activity in PsA. In comparison to RA, PsA is a more heterogeneous disease with additional features such as psoriasis, enthesitis, dactylitis, and spondylitis; it may influence the RAPID3 scores beyond peripheral arthritis alone, and the degree to which these contributed to the scores was previously unknown ${ }^{43}$. The strong associations of RAPID3 with other PRO in PsA suggest that these additional features were adequately determined with RAPID $3^{44}$. The weighting of the global and pain scores may account for the strong association observed with overall disease effect.

Our findings are complementary to earlier work conducted on the RAPID3. Coates, et al demonstrated good responsiveness and discrimination in a clinical trial [Tight Control of Psoriatic Arthritis (TICOPA)] and an observational cohort [LongTerm Outcome in Psoriatic Arthritis Study (LOPAS II)] $]^{7,45}$. Those studies showed that RAPID3 differentiated between patients in minimal disease activity versus patients with active disease. RAPID3 also correlated with levels of disease activity, as measured by 2 validated composite measures of PsA activity, PASDAS and DAPSA ${ }^{7,45}$. Conversely, our findings differed from the study conducted by Vakil-Gilani, et al in a clinical care population (patients with psoriasis and PsA) ${ }^{46}$. Although the patients had RAPID3 scores similar to those in our study, the 

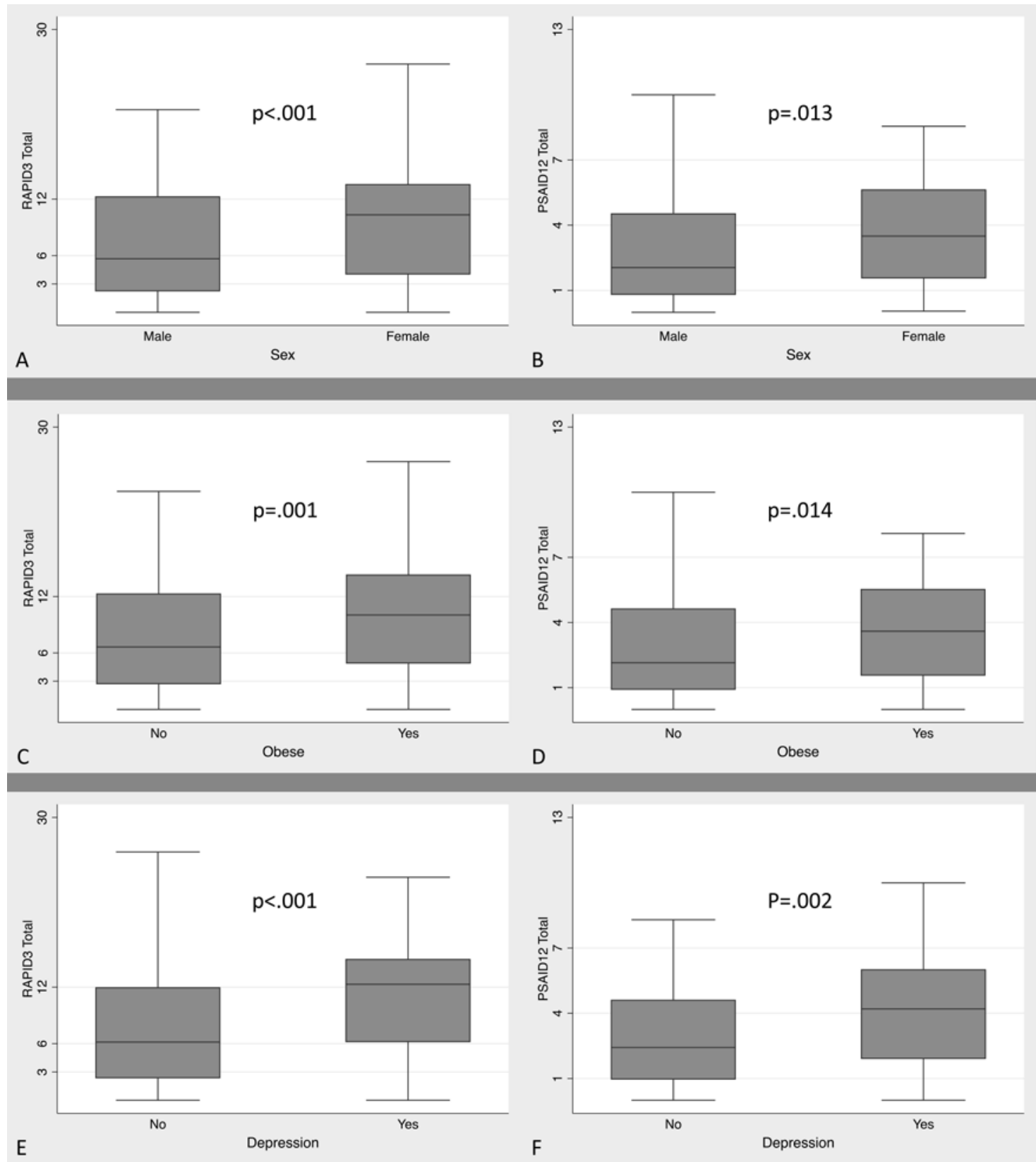

Figure 3. RAPID3 and PsAID by known subgroups: mean RAPID3 and PsAID12 scores, by sex (A, B), obesity (C, D), depression (E, F), enthesitis $(\mathrm{G}, \mathrm{H})$, and dactylitis (I, J). RAPID3 scores are significantly different among the sex, obesity, depression, and enthesitis status subgroups. PsAID scores are significantly different among the sex, obesity, and depression status subgroups. RAPID3: Routine Assessment of Patient Index Data 3; PsAID12: Psoriatic Arthritis Impact of Disease 12.

authors found a nonlinear relationship between RAPID3 and the Psoriasis Quality of Life 12-item questionnaire (PQoL-12). Based on the weak correlation between these instruments, and the inability to transform the scores, the authors concluded that RAPID3 "fails to capture mental and psychological health information" ${ }^{\prime 4}$. Our findings, however, suggest that RAPID3 was at least moderately correlated with HRQOL instruments (though PQoL12 was not measured) and was strongly correlated with PsAID.

While RAPID3 is widely used and can be used in multiple diseases, PsAID also has advantages over RAPID3: it was developed among patients with PsA for measurement of PsA and has been validated in more than 1 cohort; it includes an item addressing the skin, which RAPID3 does not directly address; and cutoffs for thresholds of meaning (i.e., patient acceptable symptom state and minimal clinically important differences) have been established. Additionally, PsAID12 was recently provisionally endorsed by OMERACT for the measurement of disease-specific HRQOL in clinical trials $^{14,43}$. Given these advantages, in particular the content 

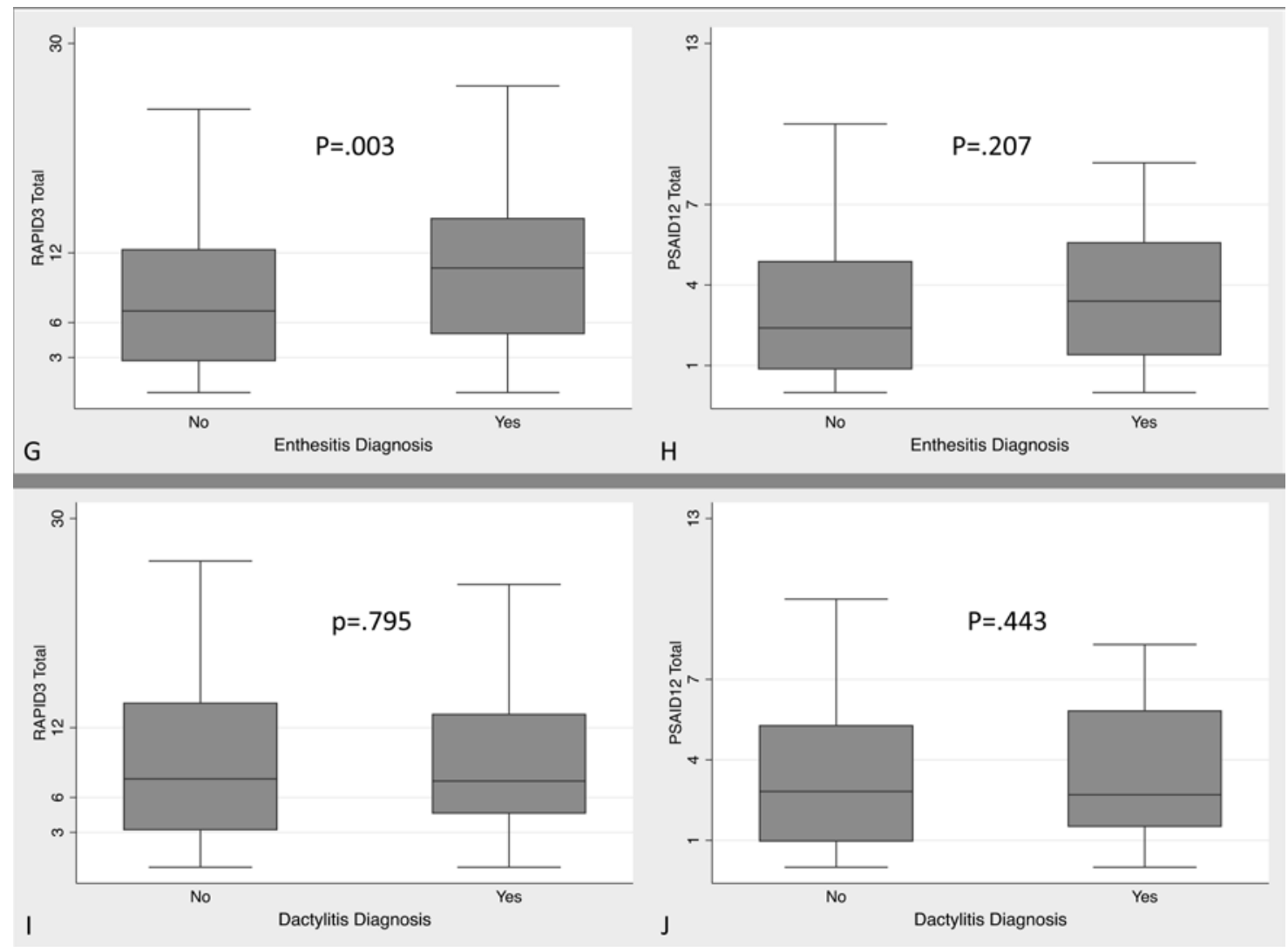

Figure 3. Continued.

validity, it is that much more interesting that the 2 measures are so strongly correlated.

Examination of contextual factors relevant for RAPID3 and PsAID in PsA is a strength of our study. Contextual factors are the other factors aside from the patient's disease that may inform the way a patient responds to a questionnaire ${ }^{26}$. This is important because these factors can be confounders that lead to differential study effects and bias ${ }^{47}$. Therefore, we assessed contextual factors to identify items that should be collected in studies to fully understand these scores. Education, insurance type, enthesitis, and total TJC were independently associated with RAPID3 scores. These same factors plus the SJC and depression were independently associated with PsAID scores. Although there may be a bidirectional relationship (e.g., reduced physical function leads to more TJC and vice versa), overall these data suggest that at a minimum these factors should be identified in observational studies using the RAPID3 or PsAID as a primary outcome. Of note, insurance type may be a marker of socioeconomic status and our findings may be related to potential undertreatment in the uninsured or underinsured psoriasis and PsA populations in the US ${ }^{48}$. Longitudinal analyses are needed to better understand the relationship between contextual factors and outcomes and whether these relationships portend poorer response.

Generalizability is a strength of our study because it is a multicenter observational cohort study representing different geographic areas in the US: 4 cities in 3 regions with varied patient populations and a wide range of disease activity and disease features, allowing for analyses in subsets of patients. We have demonstrated that using pooled data from multiple centers is possible when data collection is standardized with this PARC cohort, and longitudinal studies in the future are planned within this cohort.

This is a cross-sectional, complete case analysis and therefore has limitations. First, the study was performed in academic medical centers, so selection bias may affect the generalizability of the results. The minimal exclusion criteria should limit the effect of selection bias. We did not evaluate the instruments' reliability. Reliability has been addressed in other studies ${ }^{12,33}$. While our findings support previous validation studies of RAPID3 and PsAID, longitudinal data on discrimination, responsiveness, and construct validity of these PRO in PsA are still needed. Further, we used a conversion method to transform the scores of HAQ-DI to multidimensional HAQ from 1 center that did not collect RAPID3 but instead collected HAQ-DI, patient global, and patient pain assessment. This conversion method has previously been validated in RA (not in a PsA population) and therefore could affect the validity of our results. Excluding this center from the analysis did not affect the results. Additionally, studies to determine whether these scores can be transformed from one to another would be helpful.

Our findings demonstrate that RAPID3 and PsAID12 are strongly correlated and appear to measure similar constructs,

Personal non-commercial use only. The Journal of Rheumatology Copyright @ 2020 . All rights reserved. 
Table 3. Univariable and multivariable associations with RAPID-3 scores.

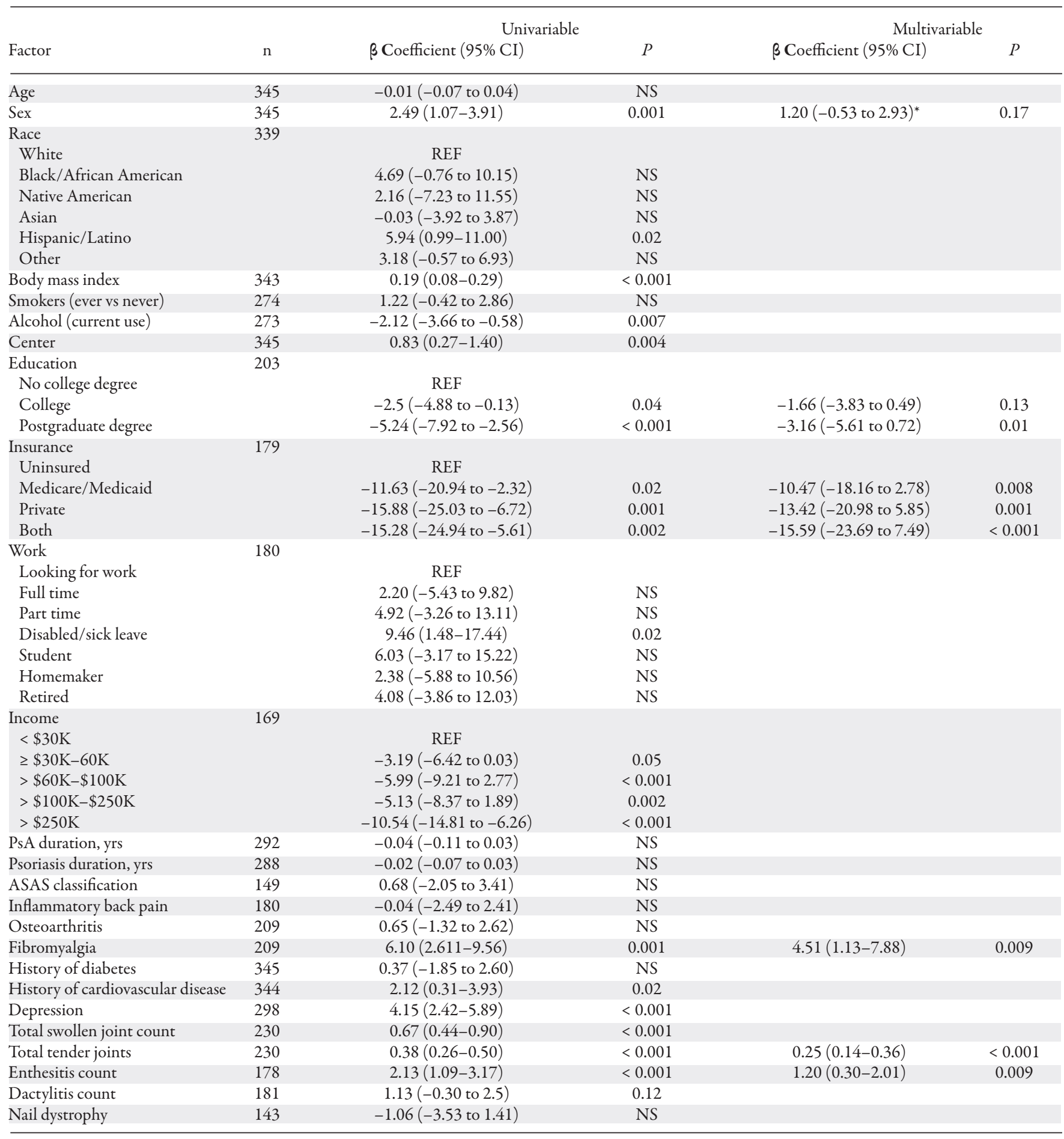

${ }^{*}$ Sex was retained in the final model as it was felt to be biologically relevant despite not being significant. The $\beta$ coefficients can be interpreted as the difference in the mean PRO score between the 2 groups. The 95\% CI do not include 0 (no difference). CI that do not cross 0 indicate statistical difference. Every increase in year of age results in an inverse relationship with the RAPID3 score. RAPID3: Routine Assessment of Patient Index Data 3; NS: not significant; PsA: psoriatic arthritis; ASAS: Assessment of Spondyloarthritis international Society; PRO: patient-reported outcomes.

broadly assessing the effect of PsA on function and wellness. The main advantages include that RAPID3 and PsAID12 are easy to complete and score and exist in both paper and mobile applications. Moreover, they are nonproprietary for clinical use. Additionally, the RAPID3 is the most commonly used PRO in clinical practice in the US ${ }^{42}$. RAPID3 and PsAID scores are 
influenced by enthesitis, joint counts, insurance type, and depression, and these contextual factors and active disease domains should be considered when interpreting the scores. Additional longitudinal studies are needed to determine which is most optimal for monitoring long-term outcomes.

\section{ACKNOWLEDGMENT}

We thank Kathleen Bush, Christina Burgese, Samantha Jackson, and Brenda Northop for their administrative support. Additionally, we thank the patients who spent time completing these assessments.

\section{ONLINE SUPPLEMENT}

Supplementary material accompanies the online version of this article.

\section{REFERENCES}

1. Ader D. Introduction: Developing the patient-reported outcomes measurement information system (PROMIS). Medical Care 2007;45:S1-2.

2. Deshpande PR, Rajan S, Sudeepthi BL, Abdul Nazir CP. Patient-reported outcomes: a new era in clinical research. Perspect Clin Res 2011;2:137-44.

3. Egleston BL, Miller SM, Meropol NJ. The impact of misclassification due to survey response fatigue on estimation and identifiability of treatment effects. Stat Med 2011;30:3560-72.

4. Orbai AM, Ogdie A. Patient-reported outcomes in psoriatic arthritis. Rheum Dis Clin North Am 2016;42:265-83.

5. Pincus T, Yazici Y, Castrejon I. Pragmatic and scientific advantages of MDHAQ/ RAPID3 completion by all patients at all visits in routine clinical care. Bull NYU Hosp Jt Dis 2012;70 Suppl 1:30-6.

6. Pincus T, Swearingen C, Wolfe F. Toward a multidimensional health assessment questionnaire (MDHAQ): assessment of advanced activities of daily living and psychological status in the patient-friendly health assessment questionnaire format. Arthritis Rheum 1999; 42:2220-30.

7. Coates LC, Tillett W, Shaddick G, Pincus T, Kavanaugh A, Helliwell PS. Value of the Routine Assessment of Patient Index Data 3 in patients with psoriatic arthritis: results from a tight-control clinical trial and an observational cohort. Arthritis Care Res 2018;70:1198-205.

8. Park SH, Choe JY, Kim SK, Lee H, Castrejon I, Pincus T. Routine assessment of patient index data (RAPID3) and Bath Ankylosing Spondylitis Disease Activity Index (BASDAI) scores yield similar information in 85 Korean patients with ankylosing spondylitis seen in usual clinical care. J Clin Rheumatol 2015;21:300-4.

9. Danve A, Reddy A, Vakil-Gilani K, Garg N, Dinno A, Deodhar A. Routine assessment of patient index data 3 score (RAPID3) correlates well with Bath Ankylosing Spondylitis Disease Activity Index (BASDAI) in the assessment of disease activity and monitoring progression of axial spondyloarthritis. Clin Rheumatol 2015;34:117-24.

10. Castrejon I, Pincus T, Wendling D, Dougados M. Responsiveness of a simple RAPID-3-like index compared to disease-specific BASDAI and ASDAS indices in patients with axial spondyloarthritis. RMD Open 2016;2:e000235.

11. Castrejon I, Bergman MJ, Pincus T. MDHAQ/RAPID3 to recognize improvement over 2 months in usual care of patients with osteoarthritis, systemic lupus erythematosus, spondyloarthropathy, and gout, as well as rheumatoid arthritis. J Clin Rheumatol 2013;19:169-74.

12. Gossec L, de Wit M, Kiltz U, Braun J, Kalyoncu U, Scrivo R, et al. A patient-derived and patient-reported outcome measure for assessing psoriatic arthritis: elaboration and preliminary validation of the Psoriatic Arthritis Impact of Disease (PsAID) questionnaire, a 13-country EULAR initiative. Ann Rheum Dis 2014;73:1012-9.

13. Orbai AM, de Wit M, Mease P, Shea JA, Gossec L, Leung YY, et al. International patient and physician consensus on a psoriatic arthritis core outcome set for clinical trials. Ann Rheum Dis 2017; 76:673-80

14. Orbai AM, Holland R, Leung YY, Tillett W, Goel N, Christensen R, et al. PsAID12 provisionally endorsed at OMERACT 2018 as core outcome measure to assess psoriatic arthritis-specific health-related quality of life in clinical trials. J Rheumatol 2019;46:990-5.

15. Di Carlo M, Becciolini A, Lato V, Crotti C, Favalli EG, Salaffi F. The 12-item Psoriatic Arthritis Impact of Disease questionnaire: construct validity, reliability, and interpretability in a clinical setting. J Rheumatol 2017;44:279-85.

16. Talli S, Etcheto A, Fautrel B, Balanescu A, Braun J, Canete JD, et al. Patient global assessment in psoriatic arthritis - what does it mean? An analysis of 223 patients from the Psoriatic Arthritis Impact of Disease (PsAID) study. Joint Bone Spine 2016; 83:335-40

17. Cauli A, Gladman DD, Mathieu A, Olivieri I, Porru G, Tak PP, et al. Patient global assessment in psoriatic arthritis: A multicenter GRAPPA and OMERACT study. J Rheumatol 2011;38:898-903.

18. Generali E, Scire CA, Cantarini L, Selmi C. Sex differences in the treatment of psoriatic arthritis: A systematic literature review. Isr Med Assoc J 2016;18:203-8

19. Nas K, Capkin E, Dagli AZ, Cevik R, Kilic E, Kilic G, et al. Gender specific differences in patients with psoriatic arthritis. Mod Rheumatol 2017;27:345-9.

20. Lubrano E, Perrotta FM, Manara M, D’Angelo S, Addimanda O, Ramonda $\mathrm{R}$, et al. The sex influence on response to tumor necrosis factor- $\alpha$ inhibitors and remission in axial spondyloarthritis. J Rheumatol 2018;45:195-201.

21. Gudu T, Etcheto A, de Wit M, Heiberg T, Maccarone M, Balanescu A, et al. Fatigue in psoriatic arthritis - a cross-sectional study of 246 patients from 13 countries. Joint Bone Spine 2016;83:439-43.

22. Braaten T, Breviu B, Walsh JA, Presson A, Zhang C, Clegg DO. Gender differences in patient reported outcomes (PROs) in psoriatic arthritis [abstract]. Arthritis Rheumatol 2015;67:3121.

23. Toy $S$, Ozbag D, Altay $Z$. The effects of pre-obesity on quality of life, disease activity, and functional status in patients with ankylosing spondylitis. North Clin Istanb 2017;4:52-9.

24. Rosas J, Llinares-Tello F, Senabre-Gallego JM, Barber-Valles X, Santos-Soler G, Salas-Heredia E, et al. Obesity decreases clinical efficacy and levels of adalimumab in patients with ankylosing spondylitis. Clin Exp Rheumatol 2017;35:145-8.

25. Micheroli R, Hebeisen M, Wildi LM, Exer P, Tamborrini G, Bernhard J, et al. Impact of obesity on the response to tumor necrosis factor inhibitors in axial spondyloarthritis. Arthritis Res Ther 2017;19:164.

26. Lee YX, Kwan YH, Png WY, Lim KK, Tan CS, Lui NL, et al. Association of obesity with patient-reported outcomes in patients with axial spondyloarthritis: a cross-sectional study in an urban Asian population. Clin Rheumatol 2017;36:2365-70.

27. Gunter T, Walsh JA. Comparison of patient and provider assesssments of response to therapy for psoriatic arthritis [abstract]. Arthritis Rheumatol 2015;67:667.

28. Sethi M, Ren-Fielding C, Caminer AC, Scher JU, Reddy SM. Clinical improvements in psoriasis and psoriatic arthritis with surgical weight loss [abstract]. Arthritis Rheumatol 2015;67:688.

29. Reddy SM, Scher JU, Swearingen CJ, Yazici Y. Gender differences in disease activity accounting for inflammatory biomarkers in psoriatic

Personal non-commercial use only. The Journal of Rheumatology Copyright $(\subset) 2020$. All rights reserved. 
arthritis routine care cohort [abstract]. Arthritis Rheumatol 2014;66:S698.

30. Taylor W, Gladman D, Helliwell P, Marchesoni A, Mease P, Mielants $\mathrm{H}$, et al. Classification criteria for psoriatic arthritis: development of new criteria from a large international study. Arthritis Rheum 2006;54:2665-73.

31. Sieper J, van der Heijde D, Landewe R, Brandt J, Burgos-Vagas R, Collantes-Estevez E, et al. New criteria for inflammatory back pain in patients with chronic back pain: a real patient exercise by experts from the Assessment of Spondyloarthritis international Society (ASAS). Ann Rheum Dis 2009;68:784-8.

32. Healy PJ, Helliwell PS. Measuring clinical enthesitis in psoriatic arthritis: assessment of existing measures and development of an instrument specific to psoriatic arthritis. Arthritis Rheum 2008;59:686-91.

33. Pincus T, Swearingen CJ, Bergman M, Yazici Y. RAPID3 (Routine Assessment of Patient Index Data 3), a rheumatoid arthritis index without formal joint counts for routine care: proposed severity categories compared to disease activity score and clinical disease activity index categories. J Rheumatol 2008;35:2136-47.

34. Anderson J, Sayles H, Curtis JR, Wolfe F, Michaud K. Converting Modified Health Assessment Questionnaire (HAQ), Multidimensional HAQ, and HAQII scores into original HAQ scores using models developed with a large cohort of rheumatoid arthritis patients. Arthritis Care Res 2010;62:1481-8.

35. Helliwell PS, Waxman R. Modification of the Psoriatic Arthritis Disease Activity Score (PASDAS). Ann Rheum Dis 2018;77:467-8.

36. Streiner D, Norman G. Validity. Health measurement scales: a practical guide to their development and use. New York: Oxford University Press; 2008:247-76.

37. The BMJ. Correlation and regression. 2019. [Internet. Accessed February 10, 2020.] Available from: www.bmj.com/about-bmj/ resources-readers/publications/statistics-square-one/ 11-correlation-and-regression

38. Walsh JA, Willinger C, Husni ME, Reddy SM, Scher JU, Ogdie A. Construct validity of RAPID3 for measurement of disease activity in psoriatic arthritis [abstract]. Arthritis Rheumatol 2017;69:1805.

39. Mokkink LB, Prinsen CA, Bouter LM, Vet HC, Terwee CB. The consensus-based standards for the selection of health measurement instruments (COSMIN) and how to select an outcome measurement instrument. Braz J Phys Ther 2016;20:105-13.
40. Mokkink LB, Terwee CB, Knol DL, Stratford PW, Alonso J, Patrick DL, et al. The COSMIN checklist for evaluating the methodological quality of studies on measurement properties: a clarification of its content. BMC Med Res Methodol 2010;10:22.

41. The Outcome Measures in Rheumatology (OMERACT) handbook. 2019 [Internet. Accessed February 10, 2020.] Available from: omeracthandbook.org

42. Pincus T, Furer V, Keystone E, Yazici Y, Bergman MJ, Luijtens K. RAPID3 (Routine Assessment of Patient Index Data 3) severity categories and response criteria: similar results to DAS28 (Disease Activity Score) and CDAI (Clinical Disease Activity Index) in the RAPID 1 (Rheumatoid Arthritis Prevention of Structural Damage) clinical trial of certolizumab pegol. Arthritis Care Res 2011;63:1142-9.

43. Michelsen B, Fiane R, Diamantopoulos AP, Soldal DM, Hansen IJ, Sokka T, et al. A comparison of disease burden in rheumatoid arthritis, psoriatic arthritis and axial spondyloarthritis. PLoS One 2015;10:e0123582.

44. Husted JA, Tom BD, Schentag CT, Farewell VT, Gladman DD. Occurrence and correlates of fatigue in psoriatic arthritis. Ann Rheum Dis 2009;68:1553-8.

45. Coates LC, Moverley AR, McParland L, Brown S, Navarro-Coy $\mathrm{N}$, O'Dwyer JL, et al. Effect of tight control of inflammation in early psoriatic arthritis (TICOPA): A UK multicentre, open-label, randomised controlled trial. Lancet 2015;386:2489-98.

46. Vakil-Gilani KM, Dinno A, Rich-Garg N, Deodhar A. Routine assessment of patient index data 3 score and psoriasis quality of life assess complementary yet different aspects of patient-reported outcomes in psoriasis and psoriatic arthritis. J Clin Rheumatol 2018;24:319-23.

47. Sterne JA, Hernan MA, Reeves BC, Savovic J, Berkman ND, Viswanathan M, et al. ROBINS-I: a tool for assessing risk of bias in non-randomised studies of interventions. BMJ 2016;355:i4919.

48. Armstrong AW, Robertson AD, Wu J, Schupp C, Lebwohl MG. Undertreatment, treatment trends, and treatment dissatisfaction among patients with psoriasis and psoriatic arthritis in the united states: findings from the National Psoriasis Foundation Surveys, 2003-2011. JAMA Dermatol 2013;149:1180-5. 